\title{
Effects of Arrangements of Spherical Particles in the Sphere model on Rock and Soil Porosity ${ }^{1}$
}

\author{
Xueqing Wang ${ }^{\mathrm{a}}$, Zibo Miao ${ }^{\mathrm{b}}$, Junjie Yu, Chongfan Li, Yi Chen, Dongchao Xue \\ School of Mechanics \& Civil Engineering, China University of Mining \& Technology Beijing, Beijing, \\ China, 100083 \\ awxqease@163.com, 'ljmiaozibo@126.com
}

Keywords: Porosity; Soil Particle; Unit Cell; Spherical Model

Abstract. Porosity is the ratio of the volume of the medium pores to the total volume.As an important indicator of the physical properties of porous rock and soil medium, It is directly related to the physical and mechanical properties of the rock and soil in the environment, and the permeability characteristics and reservoir properties.Research on the calculation method of the porosity of rock and soil medium is of great significance. The complex characteristics in the macro of non continuous, non uniform, anisotropic and non deterministic, and so on, depend on non continuity and non determinism of microstructure of soil mass fundamentally.Under the premise of not considering the soil boundary, The soil particles are abstracted as spherical particles.Considering the unit cell,we probe different arrangement of spherical particles.Rock and soil are regarded as macro performance of arrangement with countless same unit cells. Therefore,we can calculate the porosity of the soil under various arrangements, and analyze the effects of different arrangements of soil particles on the porosity.

\section{Introduction}

The complex characteristics in the macro of non continuous, non uniform, anisotropic and non deterministic, and so on, depend on non continuity and non determinism of microstructure of soil mass fundamentally."Numerous studies show,they exhibit fractal characteristics. Therefore, fractal theory become an effective method for the study of the microcosmic and meso characteristics of rock

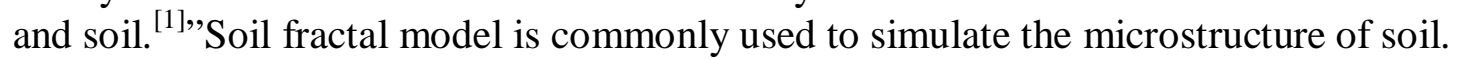

At present, there are two types of fractal models:

One kind of fractal models is Sierpinski fractal carpet.It is shown in Fig.1.

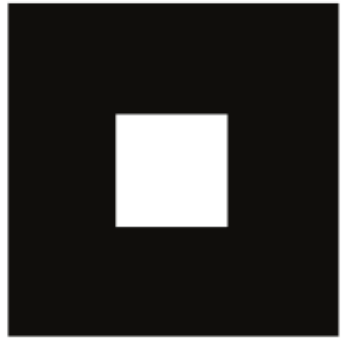

(a) $n=1$

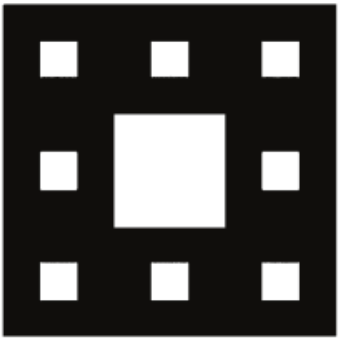

(b) $n=2$

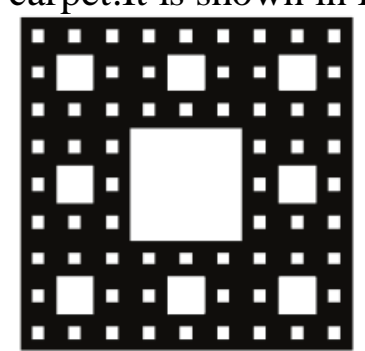

(c) $n=3$



(d) $n=4$

Fig.1 Sierpinski fractal carpet

The white parts represent the recognizable holes, whlie the black parts represent the irrecognizable holes and coexistence of carpets. With the decrease of the observation scale(That is, with the increase of $n$ ), holes with smaller aperture in the carpet are found. ${ }^{[1-3]}$ We can calculate porosity of soil using the obtained fractal dimension ${ }^{[5]}$.

Another kind of fractal model is Menger sponge model.It is shown in Fig.2.

Structure of Menger sponge fractal model:take a cube as the initial element,and divide all six sides into nine parts in average. That is to say,divide a cube into 27 parts in average. Then remove the 7 smaller cubes of the body-centered and the surface-centered,And preserve their surface. Only 20 
smaller cubes remained.Operate repeatedly $(n=1,2,3 \ldots)$, and until infinity. ${ }^{[3,4]}$ We can also calculate porosity of soil using the obtained fractal dimension ${ }^{[5]}$.

The above two models are ideal porosity model.In many cases, it is in good agreement with the actual situation ${ }^{[6]}$.However, the fractal dimension of rock and soil is often obtained by designing some complex tests. The method and accuracy of the tests are not the same exactly.Therefore,a simple model-sphere model could be used to simulate the microstructure of rock and soil.Combined with the concept of unit cell,we can utilize spherical model to realize different arrangements.And then we find the soil porosity in different arrangements and the laws of porosity with the change of Variation $\theta$.

\section{Spherical model}

After observation and analysis of the existing fractal models, It is not difficult to find that these two models both remove some smaller cubes from a bigger cube."Studies have shown that we could regard some simple entities as the calculation model of basic units,in order to imitate Mechanical behaviors of the object Approximately ${ }^{[7]}$."And the sphere is the most simple three-dimensional entity.Different arrangements which the combination of the spheres present, have a great impact on porosity of soil.

"From the images of the fresh fracture of the rock under electron microscope, we could observe that arrangements of the crystal grain include irregular point bodies, the strip bodies of different bending state, different thickness, irregular block bodies. All those constitute mineral crystal in a certain shape.These different forms of mineral--Micro Element,is a basic component of rock mass ${ }^{[9]}$."We associate unit cell with Micro element.Generally speaking, unit cells are parallelepiped.Unit cells, as the basic unit of crystal,arrange without space among cells.Unit cells are divided into three categories, including simple cubic unit cell, body centered cubic unit cell and FCC unit cell.Next, on the basis of these three kinds of unit cells(that is,three kinds of arrangements), we can calculate the porosity of the soil.(In the following calculation,porosity is represented by $\varphi$ uniformly.The radius of the spherical particle is the same, and represented by $R$ uniformly.)

Simple cubic unit cell.Fig. 3 is $3 \mathrm{D}$ graphics for simple cubic unit cell.Just as its name implies, this arrangement is the most simple one.Eight 1/8 spheres are at the cube vertex,and any two are tangent.

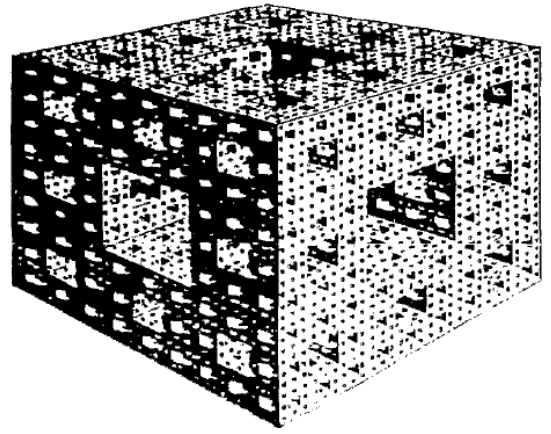

Fig.2 Menger sponge model

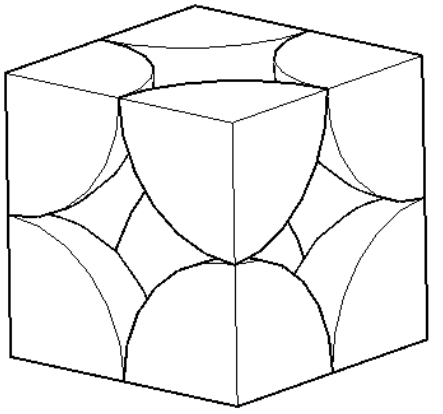

Fig.3 Simple cubic unit cell

The volume of the cube is,

$$
V=(2 R)^{3}=8 R^{3}
$$

A unit cell contains $\left(=8 \times \frac{1}{8}\right)$ sphere. (due to only $1 / 8$ sphere at every cube vertex)

The total volume of the sphere is,

$$
V^{\prime}=1 \times \frac{4}{3} \pi R^{3}=\frac{4}{3} \pi R^{3}
$$

Then, $\frac{V^{\prime}}{V}=\left(\frac{4}{3} \pi R^{3}\right) /\left(8 R^{3}\right)=\frac{\pi}{6}=0.5236$

So,the porosity is, $\varphi=1-0.5236=0.4764$

This situation is the most regular situation.And the calculated porosity is also the largest.Of course,Under the premise that any two spheres are tangent.Actually,soil particles could not contact 
with each other absolutely.So if its arrangements adopt simple cubic unit cell,the measured value may be greater than the calculated value.

Body centered cubic unit cell.Fig. 4 is 3D graphics for body centered cubic unit cell.Body centered sphere is tangent with the spheres at the cube vertex.Supposing that side of undersurface is 1 , and the angle between body diagonal and diagonal of undersurface is $\theta$.Fig. 5 shows the section of BCC unit cell.

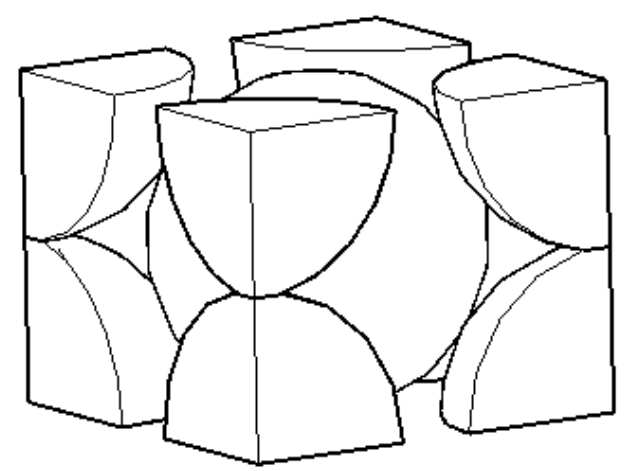

Fig.4 Body centered cubic unit cell

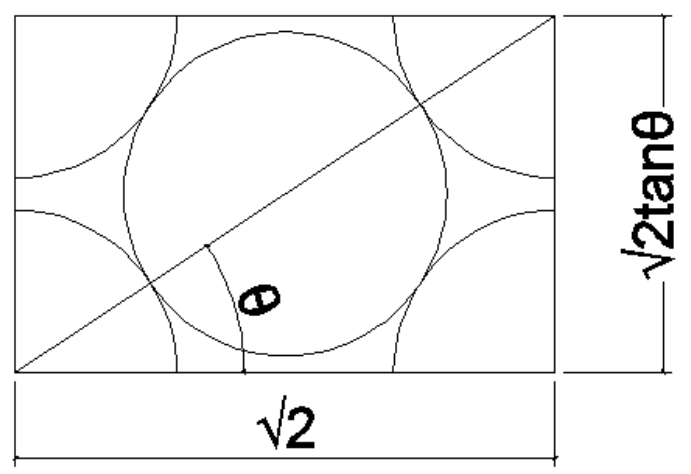

Fig.5 Section of BCC unit cell

The volume of the cube is,

$$
V=1 \times 1 \times \sqrt{2} \tan \theta=\sqrt{2} \tan \theta
$$

A unit cell contains $\chi\left(=1+8 \times \frac{1}{8}\right.$ )spheres

$$
R=\frac{\sqrt{2}}{4 \cos \theta}=\frac{1}{2 \sqrt{2} \cos \theta}
$$

To meet the conditions: $2 R \leq 1$ and $2 R \leq \sqrt{2} \tan \theta$

Therefore, $\frac{\pi}{6} \leq \theta \leq \frac{\pi}{4}$

The total volume of the sphere is,

$$
V^{\prime}=2 \times \frac{4}{3} \pi R^{3}=2 \times \frac{4}{3} \pi \times\left(\frac{1}{2 \sqrt{2} \cos \theta}\right)^{3}=\frac{\pi}{6 \sqrt{2} \cos ^{3} \theta}
$$

Then, $\frac{V^{\prime}}{V}=\frac{\frac{\pi}{6 \sqrt{2} \cos ^{3} \theta}}{\sqrt{2} \tan \theta}=\frac{\pi}{12 \cos ^{2} \theta \sin \theta}$

After calculation, it has been found that:

When $\theta=\arcsin \frac{1}{\sqrt{3}},\left(\frac{V^{\prime}}{V}\right)_{\min }=\frac{\pi}{12} \times \frac{1}{\left(\frac{\sqrt{6}}{3}\right)^{2} \times \frac{1}{\sqrt{3}}}=\frac{\sqrt{3} \pi}{8}=0.6802$

This situation corresponds to the maximum porosity, $\varphi=1-0.6802=0.3198$

In addition, when $\theta=\frac{\pi}{6}$ and $\theta=\frac{\pi}{4}$,

the corresponding porosity are, $\varphi=03019$ and $\varphi=0.2595$

For the body centered cubic unit cell,porosity is in the range of $0.25 \sim 0.35$, which makes no difference with the actual situation ${ }^{[8]}$.In order to calculate conveniently, we supposed that body centered sphere is tangent with the spheres at the cube vertex.Actually,soil particles could not contact with each other absolutely.So,the actual porosity ${ }^{[8]}$ may be bigger.

FCC unit cell. Fig.6 is 3D graphics for FCC unit cell.Face centered sphere is tangent with the spheres at the cube vertex.Supposing that radius of sphere- $R$ is 1 , and the angle between surface diagonal and the bottom edge of the cube is $\theta$.Fig.7 shows the section of FCC unit cell.

The volume of the cube is, 


$$
V=(4 \cos \theta)^{2} \times 4 \sin \theta=64 \cos ^{2} \theta \sin \theta
$$

A unit cell contains $\left.4=6 \times \frac{1}{2}+8 \times \frac{1}{8}\right)$ spheres. $R=1$,

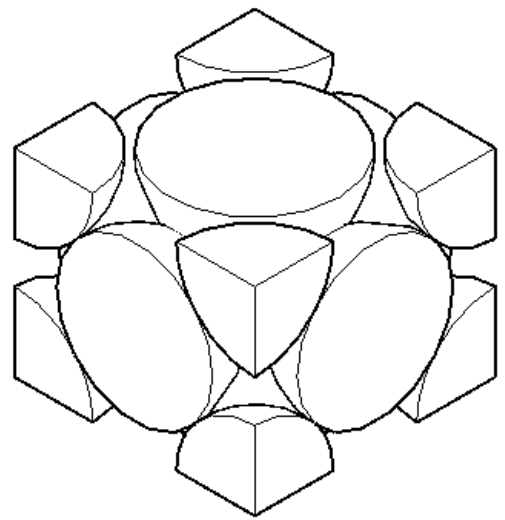

Fig.6 FCC unit cell

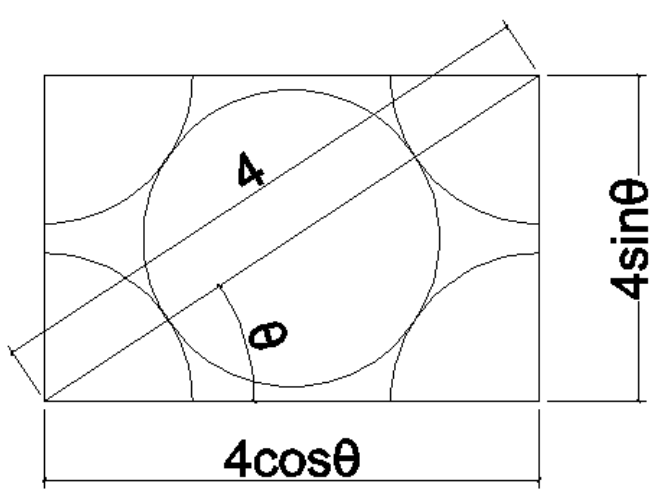

Fig.7 Section of FCC unit cell

To meet the conditions:

$$
2 R \leq 4 \sin \theta \text { and } 2 R \leq 4 \cos \theta \text { and } 4 \sqrt{2} \cos \theta \geq 4
$$

Therefore , $\frac{\pi}{6} \leq \theta \leq \frac{\pi}{4}$

The total volume of the sphere is ,

$$
V^{\prime}=4 \times \frac{4}{3} \pi \times 1^{3}=\frac{16 \pi}{3}
$$

Then,

$$
\frac{V^{\prime}}{V}=\frac{\frac{16 \pi}{3}}{64 \cos ^{2} \theta \sin \theta}=\frac{\pi}{12 \cos ^{2} \theta \sin \theta}
$$

It is no difficulty to find that Eq. 6 and Eq. 9 are in the same form. The difference lies in the meaning of $\theta$.But both maximum and minimum are equal.

$$
\text { When } \theta=\arcsin \frac{1}{\sqrt{3}},\left(\frac{V^{\prime}}{V}\right)_{\min }=\frac{\pi}{12} \times \frac{1}{\left(\frac{\sqrt{6}}{3}\right)^{2} \times \frac{1}{\sqrt{3}}}=\frac{\sqrt{3} \pi}{8}=0.6802
$$

This situation corresponds to the maximum porosity, $\varphi=1-0.6802=0.3198$

In addition, when $\theta=\frac{\pi}{6}$ and $\theta=\frac{\pi}{4}$,

the corresponding porosity are, $\varphi=03019$ and $\varphi=0.2595$

Thus,although the arrangements of these two kinds of spherical particles in the unit cells vary, it almost makes on difference in porosity.porosity is also in the range of $0.25 \sim 0.35$. The laws of porosity have been found:within a reasonable range, With the increase of $\theta$,porosity increases at first then decreases rather than increase or decrease monotonically.Maximum value are obtained in a certain value. Additionally, for both sides of $\theta$ that the maximum value correspond to, the change of the right side is more obvious than the left.

\section{Conclusions}

Actually,Soil structure is very complex.For example, there is the existence of microcrack.We attempt to show the structure of the soil vividly in a simple way,and approach the actual situation by taking advantage of fractal models.In this paper,we use sphere model to quantify the problem of porosity.and get the following conclusions: 
(1) In the simple cubic unit cell,the porosity is a constant 0.4764 .

(2) In the body centered cubic unit cell and FCC unit cell,porosity is in the range of $0.25 \sim 0.35$. With the increase of $\theta$,porosity increases at first then decreases rather than increase or decrease monotonically.

(3) The above three models are ideal porosity model,so there may exist some deviation. The measured value may be greater than the calculated value.

In order to make the applicability of sphere model more widely and closer to reality.We could consider make use of the sphere particles of non equal radius to arrange, and even attempt more complex arrangements. The problem of porosity will become abundant and complex. That provides a possibility to combine sphere model with the object-oriented programming.It is of great significance to solve the practical engineering problem of rock and soil.That is our research direction in the future.

\section{References}

[1] Jiru Zhang, Gaoliang Tao, Li Huang, Lun Yuan. Rock and soil porosity model about characterization of the pore size distribution and its application [J]. Science Bulletin, 2010, Z2:2661-2670.(In Chinese)

[2] Gaoliang Tao,Jiru Zhang. The two kinds of fractal model of rock and soil mass about characterization of particle volume and particle size distribution, [J]. Science Bulletin, 2009,06:838-846.(In Chinese)

[3] Heping Xie. Fractal porosity and fractal particle of rock and soil medium[J]. Mechanics Development, 1993,02:145-164.(In Chinese)

[4] Heping Xie, Zhida Chen. Fractal geometry and fracture of rock[J]. Acta Mechanica Sinica, 1988,03:255-264.

[5] Li Huang, Jiru Zhang, Jie Zhu, Wenjing Huang. SEM analysis of soil pore and its fractal dimension on micro scale [J]. Acta Pedologica Sinica, 2008,02:207-215. (In Chinese)

[6] Yiwen Deng, Bo Xu, Riqing Xu, Xuegui Zhan, Liyang Xu, Jianyang Lu. Quantitative Analysis of Soft Clay Three-dimensional Porosity Based on SEM Image Information [J]. Journal of Earth Sciences and Environment, 2015,03:104-110. (In Chinese)

[7] Yuyong Jiao, Ganghai Huang, Xiuli Zhang. Visual modeling for 3D spherical particle for geological body model of rock engineering $[\mathrm{J}]$. Chinese Journal of Geotechnical Engineering, 2014,04:782-787. (In Chinese)

[8] Jun He, Jianyong Shi. Apparent porosity in SEM observation of microstructure of bentonite [J].Journal of Hehai University (Natural Sciences ), 2007,02:220-224. (In Chinese)

[9] Zeyun Wang, Li Liu, Baoxian Liu. Characteristics of damage evolution of micropore and microcrack in rock [J]. Chinese Journal of Rock Mechanics and Engineering, 2004,10:1599-1603. (In Chinese) 